\title{
Varying Sugars and Sugar Concentrations Influence In Vitro Pollen Germination and Pollen Tube Growth of Cassia alata L.
}

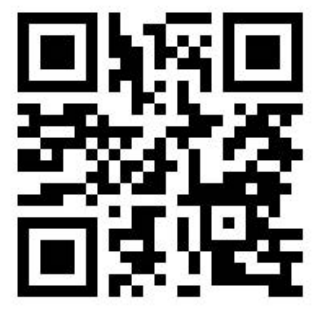

Anjela J. Lagera ${ }^{1 *}$, Lloyd O. Balinado ${ }^{1}$, John Rex Baldomeroํㅐ, Hannah Fae I. Rotairo ${ }^{1}$, Nariza L. Tero ${ }^{1}$, Mailyn S. Maghinay $^{1}$, Irma F. Baluyo ${ }^{1}$, Mary Rose Reyes ${ }^{1}$, Regine Galve ${ }^{1}$, Shellie Ann Sibao ${ }^{1}$, Jeramie V. Rufino ${ }^{1}$

This study investigates the effects of varying sugars and sugar concentrations on the in vitro germination and tube growth of pollens of Cassia alata L., a known Philippine ornamental and medicinal plant. This aims to add information on the pollination fertilization mechanism of the plant for its possible extensive cultivation. Using a pollination germination medium with different sugar concentrations (2.5, 5.0, 7.5 and $10.0 \%$ ), pollen germination and pollen tube growth is highly influenced by all sucrose concentrations and by certain glucose $(2.5 \%)$ and lactose $(2.5$ and $7.5 \%)$ concentrations. Maltose and fructose, on the other hand, are determined to be inhibitory sugars for pollen germination.

\section{INTRODUCTION}

The total count of pollen grains on a stigma usually surpasses the number required to fertilize all ovules; thus, the process of pollen growth in the carpel is highly competitive (Okusaka \& Hiratsuka, 2009). In higher plants, the elongation of pollen tube is extremely fast making the pollen tube the plant cell with the fastest growth rate. Accordingly, this swift growth of pollen tubes is essential for male reproductive success (Okusaka, \& Hiratsuka, 2009) and for the subsequent plant development.

Pollen development and tube growth (due to its high growth rate) are high energy-requiring processes (Selinski, \& Scheibe, 2014). Carbohydrates act as energy source during the two processes (Okusaka, \& Hiratsuka, 2009). The storage compounds and sugars stored in mature pollen can adequately sustain survival of pollen and germination; however, the rapid pollen tube elongation requires secretions of carbohydrates (exogenous sugars) from the stylar canal to proceed (Reinders, 2016). Exogenous sugars also provide and maintain suitable osmotic environment not only for germination of pollen but also for sustained pollen tube growth

${ }^{1}$ Department of Biological Sciences, Cavite State University, Indang, Cavite, Philippines, 4122

*To whom correspondence should be addressed: anjela_lagera@yahoo.com

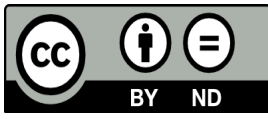

Except where otherwise noted, this work is licensed under https://creativecommons.org/licenses/by-nd/4.0/

doi:10.22186/jyi.33.1.42-45
(Baloch \& Lakho, 2001).

Most of the studies conducted on C. alata L. are on its therapeutic properties. Leaves of $C$. alata $\mathrm{L}$. contain anthraquinone derivatives which exhibit antimicrobial, antitumor, antioxidant, cytotoxic and hypoglycemic activities (Alalor, Igwilo, \& Jeroh, 2012). Crude extracts of the plant are being used to treat various skin diseases (Balinado, \& Chan, 2017) and are effective against Staphylococcus aureus and Bacillus subtilis (Alalor, Igwilo, \& Jeroh, 2012). Also, C. alata L. based soap was proven effective against opportunistic yeasts (Esimone, 2007).

Preliminary investigation of the developmental morpho-anatomy of the male gametophyte of $C$. alata $\mathrm{L}$. was already conducted (Tolentino, 2011), but limited information is known regarding its sugar metabolism and investigating this will immensely contribute to the extensive cultivation of the plant taking into consideration its medicinal properties. This study, therefore, would add light to the developmental biology of C. alata particularly to its pollen germination and pollen tube growth.

The study specifically aims to determine the effect of varying sugars and sugar concentrations on the in vitro pollen germination and tube growth of $C$. alata by calculating the germination percentage and measuring the pollen tube length after exposure to different sugars. In numerous studies on in vitro pollen germination of different plant species, sucrose exhibited strong stimulatory effects (Baloch, \& Lakho, 2001; Patel, 2017; Zhang, \& Croes, 1982), together with glucose and lactose (Ismail, 2014); thus, may also promote pollen germination in C. alata. Maltose and fructose, on the other hand, were reported to have varied effects on pollen germination of various plant species (Ismail, 2014; Okusaka, \& Hiratsuka, 2009; Nakamura, \& Suzuki, 1985). 


\section{METHODS}

\section{Pollen Collection}

Cassia alata L. flowers at anthesis were collected randomly from Cavite State University, Indang, Cavite during daytime. Flowers were immediately transported to the Department of Biological Sciences of the same institution for the conduct of the experiment. Pollen grains were collected by carefully tapping and brushing the anthers of each flower on a clean petri dish.

\section{Preparation of Pollen Germination Medium}

A Brewbaker and Kwack medium was used as pollen germination medium. It was composed of $100 \mathrm{mg} 1-1$ boric acid, $200 \mathrm{mg}$ 1-1 magnesium sulfate, 100mg 1-1potassium nitrate, 300-mg 1-1 calcium nitrate, 1\% agar and sugars (Jayaprakash, \& Sarla, 2000). Five sugars were utilized, namely; fructose, glucose, lactose, maltose and sucrose. For each sugar, four different concentrations were prepared: $2.5 \%, 5.0 \%, 7.5 \%$, and $10.0 \%$. A medium with no sugar added was used as negative control. The resulting medium was finally autoclaved to maintain sterility.

\section{Preparation of a Humid Chamber and Germination Slides}

A filter paper was placed in each petri dish before pouring distilled water sufficient enough to obtain a moist environment for the pollen. A glass slide with several (two to three) drops of hot liquid pollen germination medium at the center was then placed in each petri dish. This allowed the agar to completely cool and harden. With the aid of a nylon brush, pollen grains were transferred onto the solidified agar medium. Resulting petri dishes were then incubated in the dark for a total of three hours. This was performed in triplicate.

Observation of Pollen Germination and Pollen Tube Growth Observation for signs of pollen germination and pollen tube growth was done by microscopy thrice at one-hour interval. A single field of view per replicate that contained at least 30 solitary pollen grains was observed and photographed. A pollen grain was considered germinated when its tube length doubled the diameter of the pollen grain. The total number of pollens that germinated was determined and percent germination was calculated using the following formula.

$$
\% \text { germination }=\frac{\text { number of germinated pollens }}{\text { total number of pollens }} \times 100 \text {. }
$$

Pollen tube lengths were then measured (in $\mu \mathrm{m}$ ) with the aid of ImageJ free software using the images obtained from microscopy.

\section{Statistical Treatment}

Descriptive statistics, such as means and percentages, were utilized in determining pollen germination percentage and mean pollen tube lengths. One-way Analysis of Variance (ANOVA) was used to determine the significant differences in pollen tube growth among sugar concentrations.

\section{RESULTS}

\section{Examination of Pollen Germination}

Humid chambers containing germination slides with pollen grains were incubated for a total of three hours. The total number of germinated pollen grains per sugar concentration was obtained as shown in Figure 1. As presented, pollen grains only germinated in media containing glucose (i.e. 2.50\%) and lactose (i.e. $2.50 \%$ and $7.50 \%$ ) and all concentrations of sucrose (with $100 \%$ germination in $5.00-10.00 \%$ sucrose concentrations). On the other hand, germination was not observed in solitary pollen grains exposed to fructose and maltose.

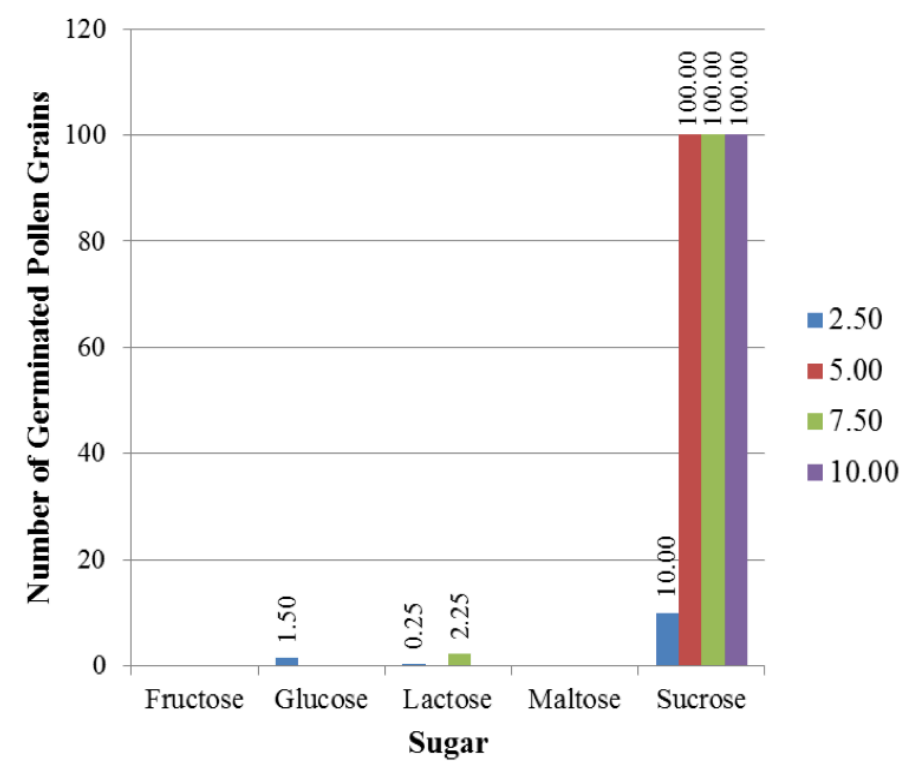

Figure 1. Mean number of germinated pollen grains per sugar concentration after $3 \mathrm{~h}$ of incubation.

As shown in Table 1 and Figure 2, pollen tube growth of C. alata L. was only observed in 2.5- and 7.5-\% lactose concentrations. Increase in pollen tube length under $7.5-\%$ concentration was found to be directly proportional to increasing time of incubation and was significantly different from other concentrations.

In addition, as presented in Table 2 and Figure 3, C. alata pollens only responded to $2.5-\%$ glucose concentration. Pollen tube lengths increased as incubation time also lengthened. No pollen tube length was observed in glucose concentrations higher than $2.5 \%$.

Varying sucrose concentrations differently influenced pollen tube growth, results were statistically significant (Table 3, Figure 4). In all concentrations, an increase in pollen tube length was observed in response to increasing time of incubation. A representative photograph of pollen tube growth on germination medium with sucrose is shown in Figure 5. 
Table 1. Mean pollen tube lengths (in $\mu \mathrm{m}$ ) in response to increasing lactose concentrations.

\begin{tabular}{|c|c|c|c|}
\hline \multirow{2}{*}{$\begin{array}{c}\text { Lactose Concentration } \\
(\%)\end{array}$} & \multicolumn{3}{|c|}{ Time (h) } \\
\hline & 1 & 2 & 3 \\
\hline 2.5 & $0.0000 \mu \mathrm{m}$ & $0.0000 \mu \mathrm{m}$ & $0.0690 \mu \mathrm{m}$ \\
\hline 5.0 & 0.0000 & 0.0000 & 0.0000 \\
\hline 7.5 & 0.0790 & 0.1500 & 0.1767 \\
\hline 10.0 & 0.0000 & 0.0000 & 0.0000 \\
\hline (-) control & 0.1351 & 0.1407 & 0.1518 \\
\hline $\begin{array}{l}F_{\text {conc }} \\
p=0.002\end{array}$ & \multicolumn{3}{|c|}{$\begin{array}{l}12.1244 \\
\text { Significant }\end{array}$} \\
\hline
\end{tabular}

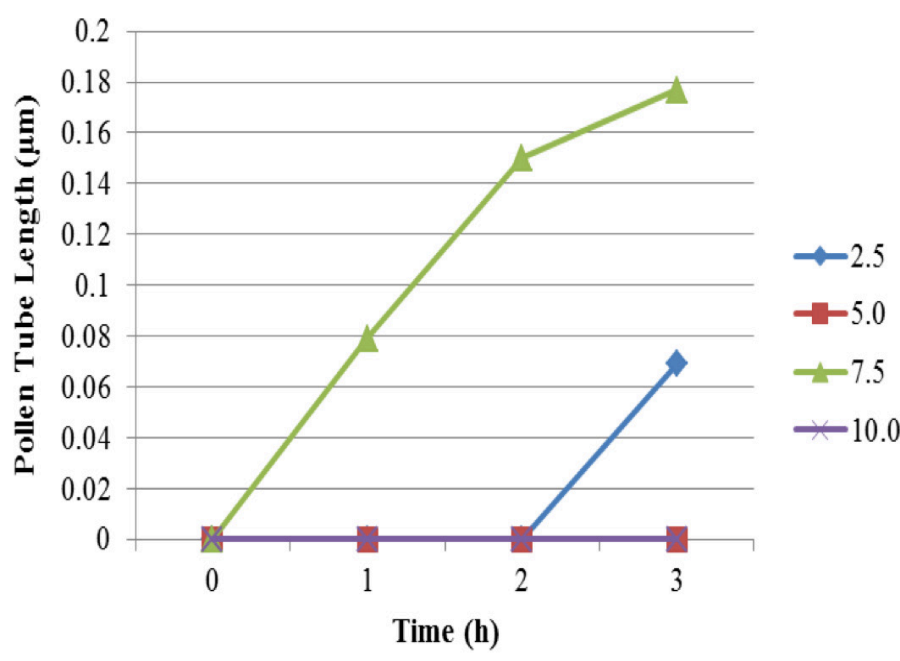

Figure 2. Graph showing mean pollen tube lengths (in $\mu \mathrm{m}$ ) in response to increasing lactose concentrations.

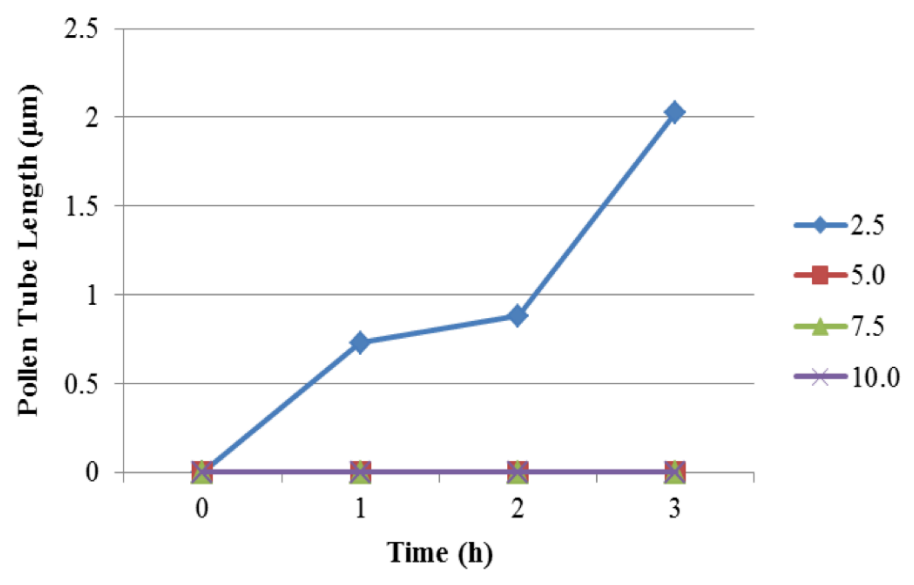

Figure 3. Mean pollen tube lengths (in $\mu \mathrm{m}$ ) in response to increasing glucose concentrations.
Table 2. Mean pollen tube lengths (in $\mu \mathrm{m}$ ) in response to increasing glucose concentrations.

\begin{tabular}{|c|c|c|c|}
\hline $\begin{array}{c}\text { Glucose Concentration } \\
(\%)\end{array}$ & \multicolumn{3}{|c|}{ Time (h) } \\
\cline { 2 - 4 } & $\mathbf{1}$ & $\mathbf{2}$ & $\mathbf{3}$ \\
\hline 2.5 & $0.7300 \mu \mathrm{m}$ & $0.8800 \mu \mathrm{m}$ & $0.0300 \mu \mathrm{m}$ \\
\hline 5.0 & 0.0000 & 0.0000 & 0.0000 \\
\hline 7.5 & 0.0000 & 0.0000 & 0.0000 \\
\hline 10.0 & 0.0000 & 0.0000 & 0.0000 \\
\hline \multicolumn{1}{|c|}{ (-) control } & 0.1351 & 0.1407 & 0.1518 \\
\hline $\begin{array}{l}F_{\text {conc }} \\
\mathrm{p}=0.007\end{array}$ & $\begin{array}{c}8.7312 \\
\text { Significant }\end{array}$ \\
\hline
\end{tabular}

Table 3. Mean pollen tube lengths (in $\mu \mathrm{m}$ ) in response to increasing sucrose concentrations.

\begin{tabular}{|c|c|c|c|}
\hline \multirow{2}{*}{$\begin{array}{c}\text { Sucrose Concentration } \\
(\%)\end{array}$} & \multicolumn{3}{|c|}{ Time (h) } \\
\hline & 1 & 2 & 3 \\
\hline 2.5 & $0.4300 \mu \mathrm{m}$ & $0.0474 \mu \mathrm{m}$ & $0.0474 \mu \mathrm{m}$ \\
\hline 5.0 & 0.0645 & 0.0701 & 0.0701 \\
\hline 7.5 & 0.0514 & 0.0613 & 0.0613 \\
\hline 10.0 & 0.0547 & 0.0582 & 0.0707 \\
\hline (-) control & 0.1351 & 0.1407 & 0.1518 \\
\hline \multicolumn{2}{|l|}{$\begin{array}{l}F_{\text {conc }} \\
p=0.006\end{array}$} & \multicolumn{2}{|l|}{$\begin{array}{c}8.6464 \\
\text { Significant }\end{array}$} \\
\hline
\end{tabular}

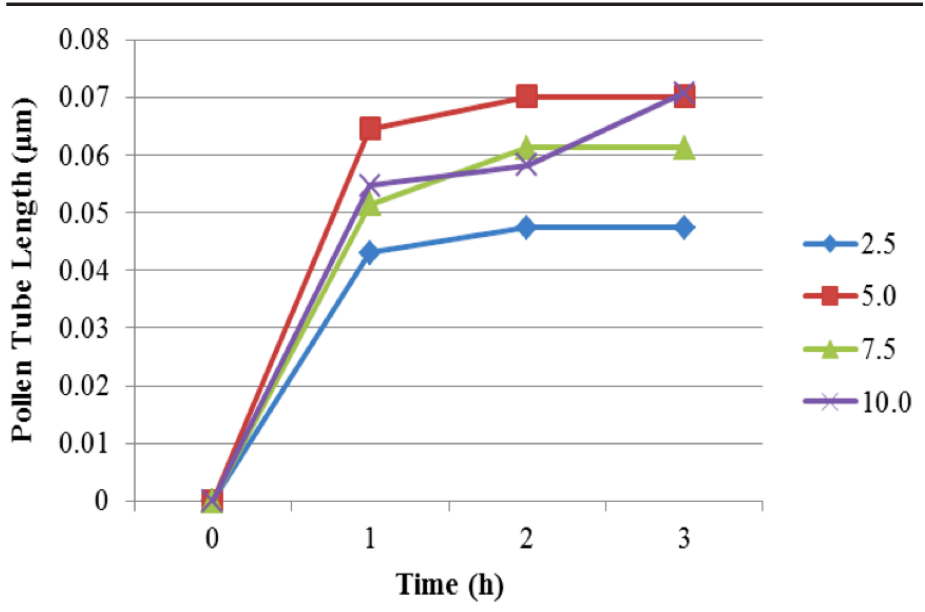

Figure 4. Mean pollen tube lengths (in $\mu \mathrm{m}$ ) in response to increasing sucrose concentrations.

\section{DISCUSSION}

Varying sugars and sugar concentrations differently influenced pollen germination and pollen tube growth of $C$. alata L. Pollens successfully germinated in the sugar sucrose and acted more effectively than glucose and lactose; while fructose and maltose strongly inhibited germination on agar medium.

The observation that glucose permitted pollen tube growth could be explained by the fact that glucose is natural pollen constitu 


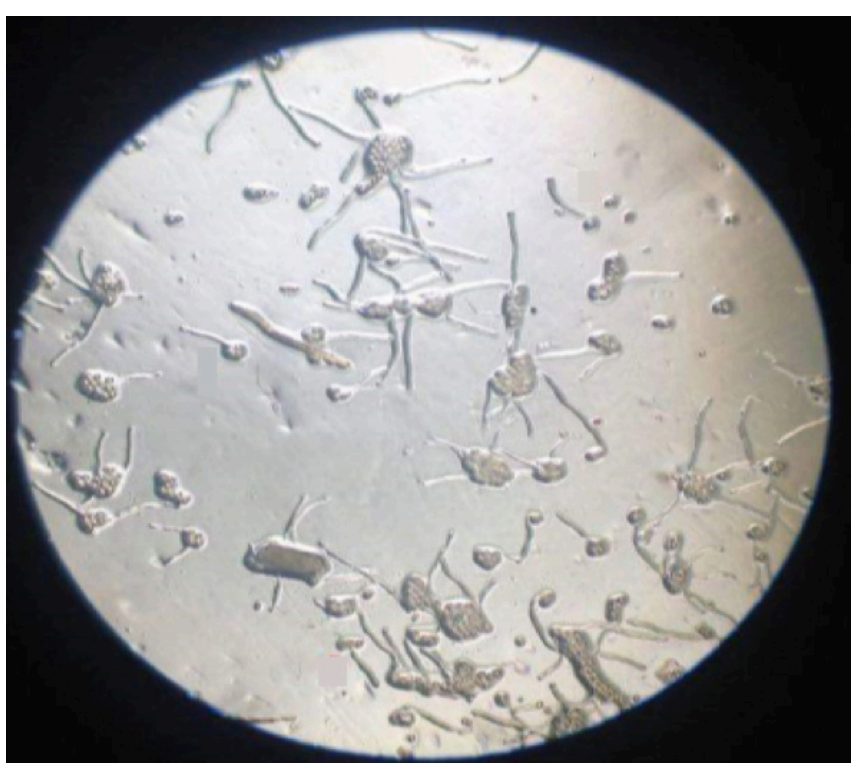

Figure 5. Pollen germination on sucrose.

ent, together with other sugars, such as arabinose and galactose (Loo \& Hwan, 1944). This sugar acts as an essential signaling molecule that controls plant growth and development and gene expression (Zhou et al., 1998). In addition, the effect of lactose in this study was similarly reported by Bishop (2009) and Ismail (2014). Most significant pollen tube growth on lactose compared to other sugars was also observed by Takao et al. (2006). Bishop (2009) even suggested that a higher concentration of lactose could be used as substitute for the normally used sucrose. The positive influence of sucrose to pollen germination and growth, on the other hand, could be attributed to the condition it provides to pollen that is similar to the condition of the stigmatic tissue of a flower; this stigma that secretes a fluid substance to rehydrate the pollen (Zhang, \& Croes, 1982). Sucrose is the most common sugar form found in the translocation stream and is transported to other nonphotosynthetic plant tissues, such as flowers, for direct metabolic use (Hopkins, \& Huner, 2009). The growth of pollen tube on sugar-free medium, in addition, could be attributed to the use of endogenous carbohydrates of the pollen without the influence-be it stimulatory or inhibitory - of other sugars present in the medium.

Similarly to the results obtained by Nakamura and Suzuki (1985), maltose strongly inhibited pollen tube growth in Camella japonica. Okusaka and Hiratsuka (2009), in addition, reported that fructose causes pollen inhibition. It was suggested that the pollen on fructose medium predominantly uses other sugars (e.g. sucrose and glucose) as respiration substrates and cannot maintain the constant level of these sugars.

This study reveals that different sugars have a considerable influence on pollen germination and pollen tube growth in $C$. alata L. Pollen tube growth is influenced by glucose, lactose and sucrose sugars; the latter being the most effective. Maltose and fructose were, on the other hand, found inhibitory of germination.
This study therefore adds information on the developmental biology of pollens of C. alata L., a known ornamental and medicinal plant in the Philippines, which can further be used for its extensive cultivation in the country.

\section{ACKNOWLEDGEMENT}

The researchers would like to acknowledge with deep and warm gratitude the Department of Biological Sciences, College of Arts and Sciences, Cavite State University for the laboratory materials and equipment used in the study.

\section{REFERENCES}

Okusaka, K., \& Hiratsuka, S. (2009). Fructose inhibits pear pollen germination on agar medium without loss of viability. Scientia Horticulturae, 122(1):51-55. doi:10.1016/j.scienta.2009.03.024

Selinski, J., \& Scheibe, R. (2014, November 3). Pollen tube growth: Where does the energy come from? Plant Signaling and Behavior, 9(12). Retrieved November 23, 2016, from doi: 10.4161/15592324.2014.977200

Reinders, A. (2016). Fuel for the road - sugar transport and pollen tube growth Journal of Experimental Botany, 67(8):2121-2123. doi:10.1093/jxb/erw113

Baloch, M. J., \& Lakho, A. R. (2001). Impact of sucrose concentrations on in vitro pollen germination of okra, Hibiscus esculentus.Pakistan Journal of Biological Sciences, 4(5):402-403.

Alalor, C., Igwilo, C., \& Jeroh, E. (2012). Evaluation of the antibacterial properties of aqueous and methanol extracts of Cassia alata. Journal of Pharmacy and Allied Health Sciences, 2(2):40-46. doi:10.3923/jpahs.2012.40.46

Balinado, L., \& Chan, M. (2017). An ethnomedicinal study of plants and traditional health care practices in District 7, Cavite, Philippines.2017 International Conference on Chemical, Agricultural, Biological and Medical Sciences (CABMS-17).doi: 10.17758/URUAE.AE0117622

Esimone, C. (2007). Evaluation of the Antiseptic properties of Cassia alata-based herbal soap.International Journal of Alternative Medicine, 6(1).

Jayaprakash, P., \& Sarla, N. (2000). Development of an improved medium for germination of Cajanuscajan (L.) Millsp.Pollen in vitro.Journal of Experimental Botany, 52(357):851-855

Loo, T., \& Hwang, T. (1944). Growth stimulation by manganese sulphate, indole3 -acetic acid and colchicine in pollen germination and pollen tube growth. American Journal of Botany, 31(6):356-367

Zhou, Li, Jang, Jang-chyun, Jones, Tamara Sheen. (April 1998). Glucose and ethylene signal transduction crosstalk revealed by an Arabidopsis glucoseinsensitive mutant. Harvard Medical School, Boston, MA.

Bishop, C. J. (2009). Pollen tube culture on a lactose medium. Stain Technology, 24(1):9-12. doi: 10.3109/10520294909139572

Ismail, O. M. (2014). In vitro germination of date palm pollen grains affected by different sugar types.Research Journal of Pharmaceutical, Biological, and Chemical Sciences, 5(1):880-886.

Takao, S., Yoshiomi, S., \& Norio, N. (2006). Japanese Journal of Palynology, 52(2):97-105

Zhang, H. Q., Croes, A. F. (1982). A New Medium for Pollen Germination in vitro. 31(1|2):113-119

Hopkins, W., \& Huner, N., 2009, Introduction to plant physiology: 4th ed, John Wiley \& Sons, Inc: USA, 503 pp.

Nakamura, N., \& Suzuki, H. (1985). Inhibition of Camellia japonica pollen tube growth by maltose.Plant and Cell Physiology, 26(6):1011-1018

Patel, E. (2015). Sucrose Needs for Pollen Germination of Impatiens balsamina L. International Journal of Innovative Research in Science, Engineering and Technology, 4(10). doi:10.15680/IJIRSET.2015.0410104

Tolentino, V. (2011). A preliminary study on the developmental morpho-anatomy of the male gametophyte of Cassia alata L. Retrieved March 16, 2017, from http://rcw-portal.ateneo.edu/rvp/details.php?id=2011-C0393 